\title{
Study the Spectral Properties of Coumarine -47 Dissolved in Chloroform
}

\author{
Rafah Abdul Hadi Omer* Mohammed T. Hussian ** \\ Lamia Kand Kalif*
}

Received 20, December, 2012

Accepted 11, March, 2014

\begin{abstract}
:
The spectral propetties (absorption and fluorescence) of Coumarine-47 laser dye have been studied. This type of laser dye belong the Coumarine family and it has dissolved in chloroform at different concentrations $(1 \times 10-5,5 \times 10-5$, and $1 \times 10-4 \mathrm{M})$ at room temperature. The achieved results have been pointed out to increase in the absorption and fluorescence as the concentration increased which are agreements with Beer - Lambert law. These have been also showed an expansion in the spectral range of absorption and fluorescence with a noticed shift in the direction of longer wavelength (Red-shift) with increasing concentration.

The quantum efficiency of the dissolved $\mathrm{C}_{47}$ in chloroform has been computed by using the brevious concentrations and their results are as follows $(69 \%, 63 \%$, and 45\%) respectively. The radiative and fluorescence lifetime have been also computed as given $(6.47 \mathrm{~ns}, 4.67 \mathrm{~ns}$, and $2.06 \mathrm{~ns})$ and $(4,07 \mathrm{~ns}, 3.22 \mathrm{~ns}$, and $1.47 \mathrm{~ns})$ respectively.
\end{abstract}

\section{Key words: Coumarin dye, Dye solutions, Spectroscopic properties.}

\section{Introduction:}

The first demonstration of a dye laser, where the active medium was a solution of dye dissolved in solvent, was reported in 1966 by Sorokin and Lankard[1]. Since then, stimulated emission from many classes of organic compounds has been reported. Some of the most popular and heavily investigated are the rhodamines and coumarins. Lasers dyes are attractive in many respects as lasing media. Their stimulated emissions are tunable throughout their broad fluorescence spectrum. Laser dyes can be used in solution with appropriate solvents or doped into a solid such as the common polymer, polymethylmethacrylate (PMMA). This level of functionality allows their concentration in the host material, and hence their absorption and gain properties, to be easily controlled. Finally, the cost of the active medium, organic dyes, is negligibly small compared to that of inorganic solidstate lasers.

Solid-state dye lasers, first demonstrated by Soffer and McFarland in 1967[2] have many advantages over their liquid counterparts. They are nontoxic, nonflammable, and can be engineered to be rugged and compact by eliminating the dye flow hardware that is necessary in a liquid system. Liquid dye lasers rely on dye flow through the laser cavity to avoid thermal problems and the buildup of photodegraded dye molecules which decrease the lasing efficiency. Dye flow equipment is often bulky and complex. Because of photodegradation, or the need to change the output wavelength range, it is necessary for the dye solution to be changed periodically. As a consequence, significant time must be spent on laser maintenance, and the user may be exposed to large quantities of toxic solvents and dyes. Solid-state

\footnotetext{
*University of Baghdad . college of science for women - physics department

**University of Baghdad . college of science - physics department
} 
dye lasers do not have a liquid gain media, so liquid circulating equipment is avoided and thermal problems are more easily compensated because there is no convection. Several solid host materials have been studied with polymer matrices being the favorite.

Unfortunately, today's popular organic laser dye molecules experiences irreversible photodegradation during optical pumping. Although laser-dyes have been under use and investigation for decades, many details of the processes responsible for dye stability and maintenance of their lasing properties from photodegradation under high pump repetition rates are still vague. Some explanations suggest the formation of dimers [3] (two mutually bonded dye molecules) or thermal breakdown due to local heat accumulation[4]. In either case, the degradation effect renders dye molecules incapable of contributing to stimulated emission. These degraded molecules can also reabsorb stimulated emission from nondegraded molecules, worsening the effect. The result is that lasing efficiency continuously and permanently decreases over a relatively short time, rendering the dye doped-

polymer useless as a lasing media and limiting the laser's operational lifetime. In applications in which gain medium replacement or movement is not desirable, the fundamental photostability of the dye molecules is of paramount importance. Attempts have been made to delay the photodegradation process using additives in the polymer matrix $[5,6]$ or by rotating the pumped media $[7,8,9]$, thereby continuously changing the location of excitation, but to my knowledge, no laser dye doped polymer has been found that can perform with the reliability and simplicity needed for long term use in a commercial system.

\section{Theory}

Once a molecule has absorbed a photon it can emit a photon upon returning to the electronic ground state from the electronic excited state. As with absorbance, the strength of the transition is governed by the transition moment. The related peaks in turn will be the dominant peaks in the resulting spectrum. Two processes that emit light from a dye molecule are fluorescence, where the emitted photon is from the decay of excited state $S_{1}$ to the ground state $S_{0}$, and phosphorescence, which is a transition from the triplet state $T_{1}$ to the electronic ground state $S_{0}$. Most ground states are singlet due to the total spin being zero, cancelled out by pairs of electrons with opposite spin. The direct transition from $S_{0}$ to $T_{1}$ is forbidden since the value of the spin quantum number $S$ would have to change. Similarly a transition from $\mathrm{T}_{1}$ to $\mathrm{S}_{0}$ is slow due to the same reason, resulting in a slow process called phosphorescence. Triplet-triplet absorption bands tend to heavily overlap the fluorescence region. The triplet state has a long lifetime leading to a buildup of population inversion.

However since the transition from $S_{0}$ to $\mathrm{T}_{1}$ is forbidden, it would require very high dye concentrations for lasing. Fluorescence can be used at relatively low dye concentrations for laser applications. If the triplet state is at a lower energy than $S_{1}$, it then competes with the buildup of the excited $S 1$ state, lowering the quantum yield of the fluorescence [4].

Decay to the lowest level of $S_{1}$ is very quick provided that the temperature is low enough to prevent thermal excitation to a higher vibronic level within $S_{1}$. The number of excited atoms $\mathrm{N}$ where $k$ is the Boltzmann's constant is Nexcited $=N_{0} \exp$ $[\Delta E=k T]$. At room temperature $k T=$ $2.15 \times 10^{-2} \mathrm{eV}$ so the population density of the excited state will be low. 
The longevity of different excited states is important to the usefulness of a dye as a laser medium. The typical time scales for these processes are: $1 \mathrm{ps}$ from $\mathrm{S}_{2}$ to $\mathrm{S}_{1}$ (non-raditative transition), $10 \mathrm{~ns}$ from $\mathrm{S}_{1}$ to $\mathrm{T}_{1}$ (non-raditative transition), $1 \mu \mathrm{s}$ from $\mathrm{T}_{1}$ to $\mathrm{S}_{0}$ (phosphorescence) and $1 \mathrm{~ns}$ from $\mathrm{S}_{1}$ to $\mathrm{S}_{0}$ (fluorescence) (Figure 1) [5].

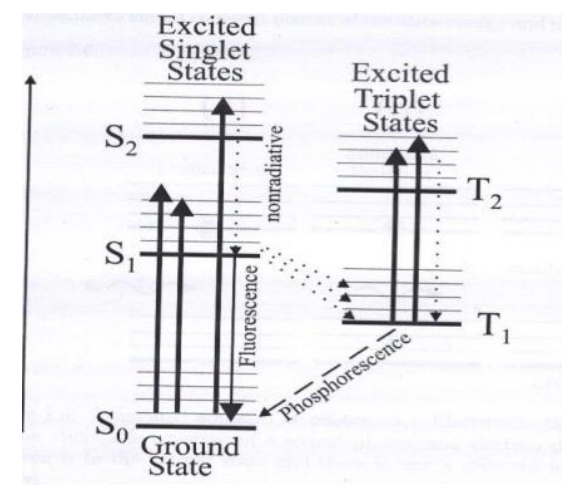

$t_{n r} \simeq 1 \mathrm{ps}$

$\mathrm{t}_{\mathrm{F}} \approx \mathrm{nns}$

$\mathrm{t}_{(\mathrm{S}-\mathrm{T}) \mathrm{nr}} \simeq 10 \mathrm{~ns}$

$\mathrm{t}_{\mathrm{P}} \simeq 1 \mu \mathrm{s}$

Fig.(1) Dye molecule energy state [5].

\section{2-1. Fluorescence}

Excitation of a molecule from absorption of a photon or thermal energy promotes the molecule to an excited energy state, setting up the possibility of emission of a photon. The excited molecules may lose energy through a non-radiative process to reach the lowest vibrational level of the excited state. Fluorescence is then the radiative loss of energy as a photon is emitted from a molecule in the excited state returning to the ground state. Since a non-radiative loss of energy occurs between the absorption and subsequent fluorescence, the emitted photon is of lower frequency than the absorbed photon producing a red shift between the absorbance and fluorescence spectra.

\section{2-2. Quantum Efficiency}

The fluorescence efficiency $\Phi_{f m}$, indicates the fraction of pump photons that are converted to fluorescence photons per dye molecule, where the maximum value is unity. Fluorescence quantum yield expresses the proportion of excited molecules that deactivate by emitting a photon[6]. The quantum yield of fluorescence is a measure of the rate of nonradiative transitions that compete with the emission of light. The knowledge of fluorescence quantum efficiency of organic dyes and its concentration dependence are essential for selecting efficient laser media [7]. The fluorescence efficiency for different concentrations of $\mathrm{C}_{47}$ in chloroform solvent was determined and their values are listed in table (1).

The spectrum of the molecular fluorescence $F(v)$ ) gives the relative fluorescence intensity at wave-number (v), this is related to the quantum efficiency by the following equation [8, $[8,9]$

$$
q_{f m}=\int_{0}^{\infty} F\left(v^{\prime}\right) d v^{\prime}
$$

In order to evaluate absolute quantum efficiency, we have to consider both the radiative and non-radiative processes taking place in the medium, therefore

$q_{f m}=\frac{K_{f n}}{K_{f m}+\Sigma K_{d}}=\frac{K_{f m}}{K_{f m}+K_{I C}+K_{I S C}}$

Where $\mathrm{K}_{\mathrm{fm}}$ fluorescence rate constant, $\mathrm{K}_{\mathrm{d}}$ nonradiative rate constant, $\mathrm{K}_{\mathrm{IC}}$ internal conversion rate constant and $\mathrm{K}_{\mathrm{ISC}}$ internal system crossing.

Since $\mathrm{K}_{\mathrm{fm}}=1 / \tau_{\mathrm{fm}}$ and $\tau_{\mathrm{f}}=1 / \mathrm{K}_{\mathrm{fm}}+$ $\Sigma \mathrm{K}_{\mathrm{d}}$

Therefore

$$
q_{f m}=\frac{\tau_{f}}{\tau_{f m}}=\int_{0}^{\infty} F\left(v^{\prime}\right) \text {------ (3) }
$$

Where $\tau_{\mathrm{f}}$ is the fluorescence lifetime and $\tau_{\mathrm{fm}}$ is the radiative lifetime of the excited state to circumscribe the possible reasons for $\mathrm{q}_{\mathrm{fm}}$ variations. The radiative lifetime being a function of the absorption strength, it is invariant 
for any molecule unless its absorption spectrum changes. As this, to first order, is not the case in concentration quenching, the mechanism for $\mathrm{q}_{\mathrm{fm}}$ variations must be changes in $\tau_{\mathrm{f}}$, caused, for example, by changes in the nonradiative lifetime of the excited state [10].

\section{Results and Discussion:}

To study the absorption spectrum for the chloroform, it can be shown from figure (2) that it has no absorption at the spectral range of $\mathrm{C}_{47}$ dye within wavelength range $(300-500 \mathrm{~nm})$. Absorption spectra of $\mathrm{C}_{47}$ dissolved in chloroform with different concentration $\left(1 \times 10^{-4}, 5 \times 10^{-5}\right.$ and $\left.1 \times 10^{-5} \mathrm{M}\right)$ are shown in figures (2) ,(3) and(4) respectively. From these figures one can deduced that $\mathrm{C}_{47}$ solution absorption spectrum has a wide spectral range at wavelength range $(300-$ $500 \mathrm{~nm})$. It has also be shown that maximum absorption appears at higher concentration at a wavelength $(393 \mathrm{~nm})$ and red shifted by $\approx 13 \mathrm{~nm}$, while at lower concentration $\left(1 \times 10^{-5} \mathrm{M}\right)$ in other word at wavelength $380 \mathrm{~nm}$ this is due to the fact that an increase in concentration produce an increase in number of molecules in volumetric unit which effect the energy state. This increase in concentration produce an increase in perturbation field of the molecules and while at the concentration decreases the relative intensity decrease which is in agreement with Beer - Lambrat law. Table (1) presents the concentration variation with the maximum absorption wavelength $\left(\lambda_{\text {abs }}\right)$ and relative intensity.

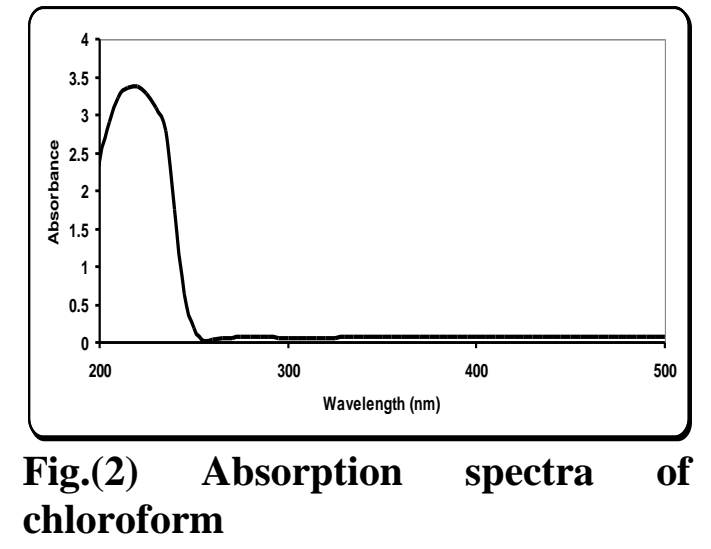

Table (1) The effect of dye concentration variation on the absorption spectra and the relative intensity

\begin{tabular}{|c|c|c|c|c|}
\hline $\begin{array}{c}\text { Concentration } \\
(\mathrm{mol} / \mathrm{liter})\end{array}$ & $\begin{array}{c}\mathrm{I}_{\text {abs }} \\
(\mathrm{a} . \mathrm{u})\end{array}$ & $\begin{array}{c}\lambda_{\max } \\
(\mathrm{abs}) \\
(\mathrm{nm})\end{array}$ & $\begin{array}{c}\Delta \lambda \\
\text { abs } \\
(\mathrm{nm})\end{array}$ & $\Delta \lambda_{\text {abs }}(\mathrm{FWHM})(\mathrm{nm})$ \\
\hline $1 \times 10^{-5}$ & 20.5 & 380 & 50 & 20 \\
\hline $5 \times 10^{-5}$ & 61.5 & 385 & 70 & 25 \\
\hline $1 \times 10^{-4}$ & 66.1 & 393 & 90 & 30 \\
\hline
\end{tabular}

The spectral properties of chloroform with $\mathrm{C}_{47}$ were studied by recording the fluorescence emission spectra for different concentrations $\left(1 \times 10^{-4}, 5 \times 10^{-5}\right.$ and $\left.1 \times 10^{-5} \mathrm{M}\right)$ as shown in figures (2),(3) and (4). The peak wavelength of fluorescence for the chloroform with $\mathrm{C}_{47}$ dye shows red shift from that for pure dye. This change may be attribute to the structure of dye, the fluorescence from the excited state dye molecule is reabsorbed by the ground state molecule which shifts the fluorescence peak to lower energies. The fluorescence bandwidth of the dye in chloroform solvent is boarder than that for pure dye, this is due to the fact that the spectral characteristics of the dye insolvent depends on the intermolecular between the dye and chloroform molecules.

The relative intensity was determined as a function of the dye concentrations and the results are tabulated in table (2).It can be shown that the highest relative intensity for the 
peak wavelength at the highest dye concentration.

Table (2) The effect of dye concentration variation on the fluorescence spectra and the relative intensity

\begin{tabular}{|l|l|l|l|l|}
\hline $\begin{array}{l}\text { Concentration } \\
(\text { mol/liter })\end{array}$ & $\begin{array}{l}\text { I flo } \\
\text { (a.u) }\end{array}$ & $\begin{array}{l}\lambda \text { max } \\
(\text { flo }) \\
(\mathrm{nm})\end{array}$ & $\begin{array}{l}\Delta \lambda \text { flo } \\
(\mathrm{nm})\end{array}$ & $\begin{array}{l}\Delta \lambda_{\text {flo }} \\
(\mathrm{FWHM}) \\
(\mathrm{nm})\end{array}$ \\
\hline $1 \times 10^{-5}$ & 18 & 402 & 30 & 25 \\
\hline $5 \times 10^{-5}$ & 44.5 & 405 & 60 & 40 \\
\hline $1 \times 10^{-4}$ & 64.6 & 410 & 70 & 45 \\
\hline
\end{tabular}

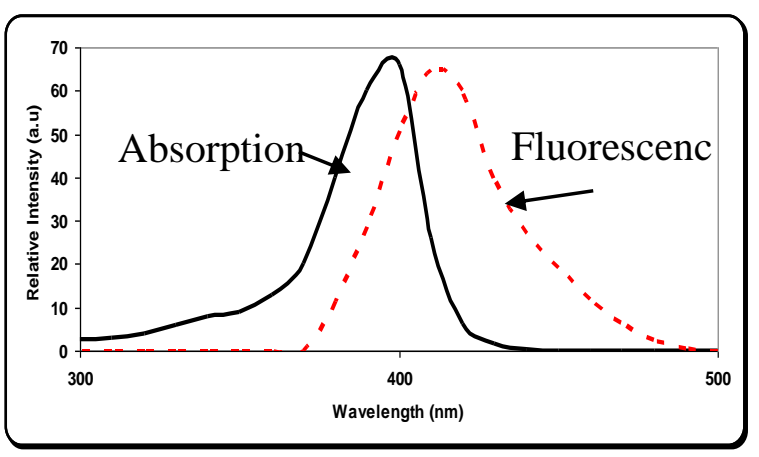

Fig. (3) Absorption and fluorescence spectra of $\mathrm{C} 47$ dissolved in chloroform at concentration $(1 \times 10-4$ M )

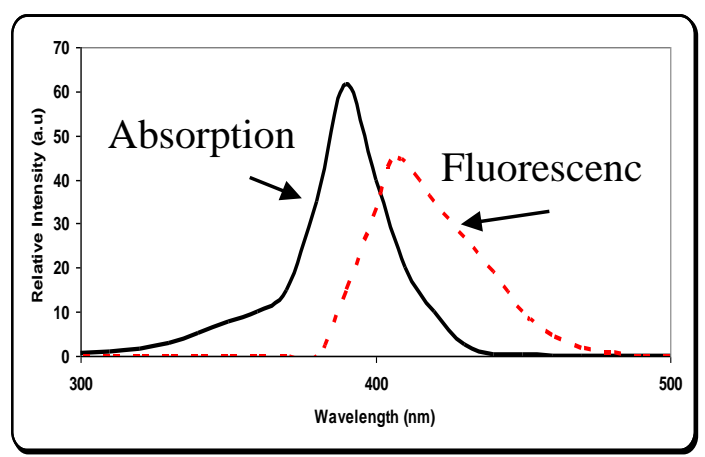

Fig. (4) Absorption and fluorescence spectra of $\mathrm{C} 47$ dissolved in chloroform at concentration $(5 \times 10-5$ M )

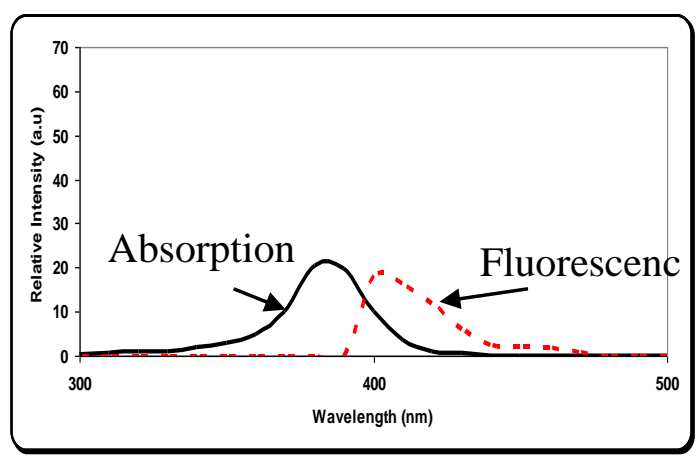

Fig. (5) Absorption and fluorescence spectra of $\mathrm{C} 47$ dissolved in chloroform at concentration $(1 \times 10-5$ M )

The fluorescence quantum yield of the dye was determined on the basis of the absorption and fluorescence spectra of the dye dissolved in chloroform. This was calculated by using the following equation:

$q_{f m}=\frac{\text { Areaunderthefluorescencespectrumcurve }}{\text { Areaundertheabsorptionspectrumcurve }}$

From the results of calculation by using computer model (Matlab 6.5) and the results tabulated in table (3).

Table (3) Represents quantum efficiencies of the $\mathrm{C}_{47}$ in chloroform solvent

\begin{tabular}{|c|c|}
\hline $\begin{array}{c}\text { Concentration } \\
(\mathrm{mol} / \mathrm{liter})\end{array}$ & $\begin{array}{c}\text { Quantum } \\
\text { efficiency } \% \mathrm{q}_{\mathrm{fm}}\end{array}$ \\
\hline $1 \times 10^{-5}$ & $69 \%$ \\
\hline $5 \times 10^{-5}$ & $63 \%$ \\
\hline $1 \times 10^{-4}$ & $45 \%$ \\
\hline
\end{tabular}

\section{Conclusion:}

From the observed results of studying the fluorescence and absorption spectra of $\mathrm{C}_{47}$ dye dissolved in chloroform one can conclude that the increase in dye concentration showed an increase in relative intensity for both spectra, which is in agreement with Beer - Lambrt law. The Stock's shift for fluorescence spectrum of the $\mathrm{C}_{47}$ dye dissolved in chloroform is toward the longer wavelength (low energy) as 
the concentration increased. It must be noted that the quantum yield of the dye solution decrease as the dye concentration increase. Finally as the dye concentration increase the interference region between the fluorescence and absorption spectra have been increased which produced a decrease in Stock's shift.

\section{References:}

[1] M. Maeda, 1984, Laser Dyes, "Properties of Organic Compounds for Dye Lasers", Academic Press, Inc., Orlando, Florida, pp. 1.

[2] K. Kuriki, T. Kobayashi, N. Imai, T. Tamura, S. Nishihara, Y. Nishizawa, A. Tagaya, Y. Koike, Y. Okamoto, 2000, Appl. Phys. Lett. 77, 331.

[3] K.H. Drexhage, 1990," Structure and Properties of Laser Dyes in Topics in Applied Physics, Dye Lasers, Springer-Verlag, Berlin, Germany, pp. 21-22.

[4] F. Amat-Guerri, A. Costela, J.M. Figuera, F. Florido, R. Sastre, 1993, Chem. Phys. Lett. 209, 352.
[5] C. Dudley,2004," Absorption, Fluorescence and Applied Spontaneous Emission of Blue - Emitted Dyes", Thesis, Washington State University.

[6] Internet, Practice 4.2,2009, Fluorescence Quantum Yield ," Fluorescence Quantum Yield of Dyes in Highlighter Pen, No. 9.

[7]C.V.Bindhu, S.S. Harilal, V.P.N. Nampoori and C.P.G. Vallabhan,1999, "Solvent Effect on Absolute Fluorescence Quantum Yield of Rhodamine 6G Determined using Transient Thermal Lens Technique", Modern Physics Letters B, Vol. 13, No. 16, 563.

[8] G. Somasundaram, A. Ramalingam, J. Photochem. Photobiol, 1999, A 125, 97.

[9] G. Somasundaram, A. Ramalingam, J. Lumin, 2000," Gain Studies of Coumarin 1 Dye - Doped Polymer Laser". 90, 5.

[10] G. Somasundaram, A. Ramalingam,2000, " Gain Studies of Coumarin 490 Dye - Doped Polymer Laser". Chem. Phys. Lett 324, 28 .

\section{دراسة الخصائص الطيفية لصبغة الكومرين -47 المذابة في مذيب الكلوروفورم لمباء قند خلبفـ

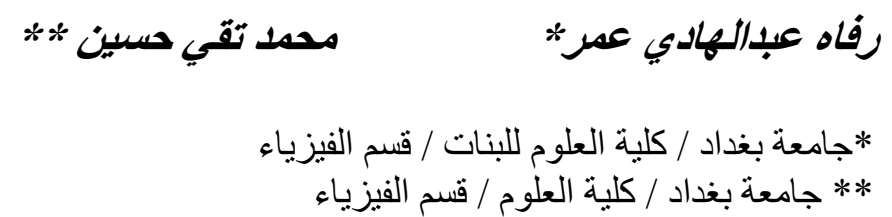

تمت دراسة الخصائص الطيفية ( الامتصاص و الفلورة) لصبغة الكومرين - 47 الليزرية. وتنتمي هذه الكئ

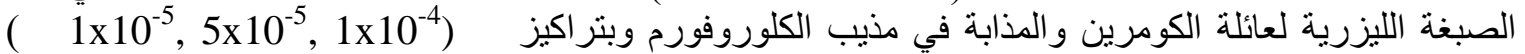

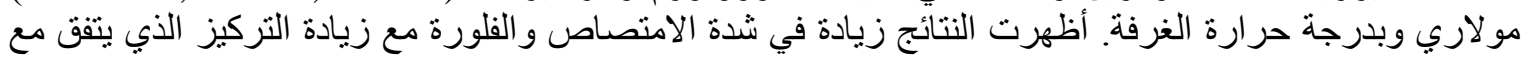

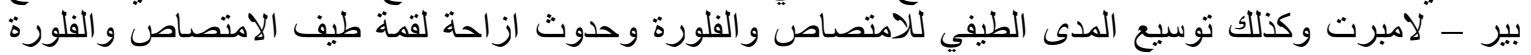

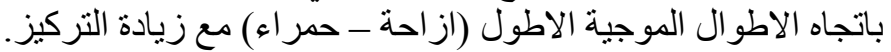

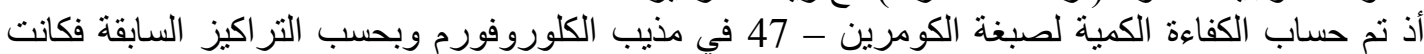
النتائج كما يلي: ( 69\% , 63\% , 63\% ) على التوالي وكذلك زمن العمر الاشعاعي ( 6.47, 4.67, 2,06 ) نانوثانية وزمن عمر الفلورة (4.07, 3.22, 1.47 ) نانوثانية و على التوالي ـ 